\title{
Opioids and an Active Population: Athletic Trainers' Perceptions
}

Dana Karlene Bates

Pacific University, dbates@pacificu.edu

Follow this and additional works at: https://nsuworks.nova.edu/ijahsp

Part of the Other Medicine and Health Sciences Commons, and the Sports Sciences Commons

\section{Recommended Citation}

Bates DK. Opioids and an Active Population: Athletic Trainers' Perceptions. The Internet Journal of Allied Health Sciences and Practice. 2020 Jan 01;18(1), Article 4.

This Manuscript is brought to you for free and open access by the College of Health Care Sciences at NSUWorks. It has been accepted for inclusion in Internet Journal of Allied Health Sciences and Practice by an authorized editor of NSUWorks. For more information, please contact nsuworks@nova.edu. 


\title{
Opioids and an Active Population: Athletic Trainers' Perceptions
}

\begin{abstract}
Purpose: Opioid use, misuse, and abuse are occurring in both high school and collegiate athletes. There is limited research that examines athletic trainers' perceptions of opioids. The purpose of this qualitative research study was to investigate athletic trainers' awareness and practice of opioids in an active population. Method: Qualitative inquiry was used to explore athletic trainers' awareness of opioids in an active population. Ten athletic trainers ( 7 male, 3 female) employed in the Northwest Athletic Training Association (6 collegiate, 4 high schools) volunteered for this study. Phone interviews were conducted in July to September 2017 with a semi-structured interview protocol. Interview data were analyzed inductively to uncover dominant themes, first by organizing the data, then summarizing into codes, and finally interpreting. Trustworthiness included both peer review and member checks. Results: Three themes emerged from this study regarding athletic trainers' practice with opioids: 1) athletic trainers have a responsibility to their patients regarding opioids, 2) communication with patients about opioids, and 3) athletic trainer perceived a lack of education regarding opioids. Conclusions: Evidence demonstrated that athletic trainers feel they have a role with opioids and their patients. While the majority of participants in this study did discuss opioids with their patients, athletic trainers felt they were lacking knowledge of opioids. Future research should explore athletic trainers' knowledge and education surrounding opioids and an active population.
\end{abstract}

\section{Author Bio(s)}

Dana K Bates, PhD, ATC, is an Assistant Professor and Coordinator of Clinical Education for the Athletic Training program at Pacific University. 


\title{
1IVAHSP \\ The Internet Joumnal of Allied Health Sciences and Practice \\ Dedicated to allied health professional practice and education Vol. 18 No. 1 ISSN 1540-580X
}

\section{Opioids and an Active Population: Athletic Trainers' Perceptions}

\author{
Dana Karlene Bates \\ Pacific University \\ United States
}

\begin{abstract}
Purpose: Opioid use, misuse, and abuse are occurring in both high school and collegiate athletes. There is limited research that examines athletic trainers' perceptions of opioids. The purpose of this qualitative research study was to investigate athletic trainers' awareness and practice of opioids in an active population. Method: Qualitative inquiry was used to explore athletic trainers' awareness of opioids in an active population. Ten athletic trainers (7 male, 3 female) employed in the Northwest Athletic Training Association ( 6 collegiate, 4 high schools) volunteered for this study. Phone interviews were conducted in July to September 2017 with a semi-structured interview protocol. Interview data were analyzed inductively to uncover dominant themes, first by organizing the data, then summarizing into codes, and finally interpreting. Trustworthiness included both peer review and member checks. Results: Three themes emerged from this study regarding athletic trainers' practice with opioids: 1) athletic trainers have a responsibility to their patients regarding opioids, 2) communication with patients about opioids, and 3) athletic trainer perceived a lack of education regarding opioids. Conclusions: Evidence demonstrated that athletic trainers feel they have a role with opioids and their patients. While the majority of participants in this study did discuss opioids with their patients, athletic trainers felt they were lacking knowledge of opioids. Future research should explore athletic trainers' knowledge and education surrounding opioids and an active population.
\end{abstract}

Keywords: prescription pain medication, pain management, athlete 


\section{INTRODUCTION}

Athletic trainers work with patients who frequently are involved in activities that can result in injury and pain. Patients may seek or be given opioids to alleviate pain. Assisting and helping our patients manage associated pain related to their injuries is critical when considering the well-being of our patients. While there has been a strong emphasis on chronic pain and misuse and abuse of opioids, one may question if high school students may be at risk. With data from the 2011 Monitoring the Future, McCabe, West and Boyd reported that $8.7 \%$ of 12 th graders indicated using prescription opioids without a doctor's orders during the past year. ${ }^{1}$ Also using data from the Monitoring the Future, Miech et al found that opioid use before high school graduation was independently associated with a $33 \%$ increase in the risk of future opioid misuse after high school. ${ }^{2}$ These data demonstrated that high school students are at risk of developing the use and abuse of opioids. ${ }^{1,2}$

Athletic trainers work with an active population, and data also demonstrated that adolescents who participate in sport may also be at a higher risk for opioid use and abuse..$^{3-6}$ Veliz et al investigated undergraduate students and involvement in sports and found an association between previous involvement in sports and prescription opioid use and misuse. ${ }^{3}$ In a Secondary Student Life Survey, Veliz et al found that male adolescents who participated in organized sports had higher odds of being prescribed an opioid medication during the past year and higher odds of past-year medical misuse of opioid medication over those who did not patriciate in organized sport. ${ }^{4}$ In another study, nonmedical use of prescription opioids was investigated among adolescents who participated in competitive sports, and it was found that those who participate in football or wrestling had nearly a $50 \%$ increase in odds of nonmedical use of prescription opioids as compared with their peers who did not participate in these sports. ${ }^{5}$ The National Collegiate Athletic Association (NCAA) conducted a study on student-athletes and determined that in collegiate athletics, $23 \%$ of student athletes reported using pain medication in the past year, and a majority of those who reported use had a prescription for the medication. ${ }^{6}$ However, $6 \%$ of collegiate athletes reported using an opioid without a prescription in the prior year. ${ }^{6}$ These findings present critical information for health care providers about the potential risk associated with sports participation and the use and misuse of prescription drugs.

As research has demonstrated, opioids use, misuse, and abuse are of concern in high school and collegiate athletics. ${ }^{1-6}$ These findings have suggested the need for greater awareness about opioids in an active population for athletic trainers, coaches, and parents. Opioid drug misuse and abuse have reached a point at which the President of the United States declared the opioid crisis a public health emergency in October 2017.7 As health care providers, athletic trainers are often in the forefront for acute pain management and are critical in educating patients on the management of pain. The purpose of this study was to investigate athletic trainers' awareness and practice of opioids.

\section{METHODS \\ Research Design}

A phenomenological approach was used to explore the experiences of athletic trainers and was used in this study because the aim was to further understand athletic trainers' awareness and practice of opioids. ${ }^{8}$ Phone interviews with athletic trainers was used for participants to express thoughts about opioids and their patients. Participants responses remained confidential The interview protocol and consent form were reviewed and approved by the Institutional Review Board. All participants signed a consent form before phone interviews.

\section{Participants}

Recruiting participants took place through purposeful sampling procedures. The sample was reflective of the following criteria: 1) certified athletic trainers who were not students, 2) employed in either a high school or college/university, and 3) employed in District 10. To isolate the inclusion criteria, e-mail addresses of potential participants were collected through the National Athletic Training Association. The principal investigator chose these inclusion criteria in order to capture certified athletic trainers' awareness and practice of opioids in an active population in District 10. An e-mail was sent to potential research participants in July 2017 asking them to participate in a study investigating awareness of opioids. Participants were also informed they would receive a $\$ 25$ gift card as an incentive to participate. Ten athletic trainers from District 10 (7 male, 3 female) who were employed in either collegiate $(n=6)$ or high school $(n=4)$ setting and held a master's $(n=6)$ or bachelor's $(n=4)$ degree volunteered for this study (see Table 1). Participants were on average 36.4 years old with 14.1 years of experience as an athletic trainer. They had been in their current position for an average of 7.8 years. Theoretical saturation up to the point in which additional data provided no new insight into the research questions was met at 10 participants as the principal investigator heard the same comments again and again at which time no further interviews were conducted. ${ }^{8}$

(C) The Internet Journal of Allied Health Sciences and Practice, 2020 
Table 1. Study Participants

\begin{tabular}{ccccccc}
\hline Participant & Sex & Clinical Setting & Age & $\begin{array}{c}\text { Highest } \\
\text { Degree }\end{array}$ & $\begin{array}{c}\text { Years of } \\
\text { Experience }\end{array}$ & $\begin{array}{c}\text { Years at } \\
\text { Current }\end{array}$ \\
\hline 1 & Male & High School & 26 & MS & 4 & 2 \\
2 & Male & High School & 27 & MS & 5 & 2 \\
3 & Female & Collegiate & 29 & BS & 6 & 1 \\
4 & Female & Collegiate & 26 & MS & 4 & 1 \\
5 & Female & High School & 37 & BS & 17 & 15 \\
6 & Male & Collegiate & 50 & MEd & 28 & 23 \\
7 & Male & Collegiate & 42 & MS & 20 & 11 \\
8 & Male & High School & 35 & BS & 11 & 14 \\
9 & Male & Collegiate & 44 & BA & 21 & 4 \\
\hline 10 & Male & Collegiate & 48 & MS & 25 & \\
\hline
\end{tabular}

\section{Data Collection Procedures}

Data were collected through semi-structured phone interview in July through September 2017. Semi-structured, open-ended questions were used for individual responses and gave an opportunity to ask more probing or follow-up questions if further clarification was necessary. ${ }^{8}$ Phone interviews were also more practical for this study than in-person interviews because of the athletic trainers' locations, which spanned District 10. The questions were designed to discover the athletic trainers' awareness and practice of opioids while working with an active population (see Table 2). Participants were asked to further clarify or expand on any answers that were a yes or no statement. The principal investigator developed the interview protocol based on previous experience with qualitative research. Interview questions were also peer-reviewed by an experienced qualitative researcher.

Phone interviews on average were 25 minutes. These interviews were recorded using H4n Pro (version 4.0, Zoom, Hauppauge, NY).

Table 2. Semi Structured Interview Questions
Questions
Elaboration
1. What do you know about opioids?
2. What are your thoughts around opioids?
3. Are you aware of any of your current or previous athletes taking opioids?
If yes, can you elaborate?
4. Are you aware of any of your current or previous athletes abusing opioids?
If yes, can you elaborate?
5. Are you aware of any of your current or previous athletes having addictions to
If yes, can you elaborate? opioids?
6. Do you believe there is an opioid crisis?
If yes, can you elaborate?
7. If you did have an athlete who came back from surgery and was prescribed opioids If yes, can you elaborate? would you discuss anything with them?
8. Does your school/district have a specific policy addressing opioids? If yes, can you elaborate?

Interviews were recorded and replayed for transcription for all research participants, and interviews and information were stored

(C) The Internet Journal of Allied Health Sciences and Practice, 2020 
on a password-protected personal laptop. The principal investigator transcribed all interviews. All participants received a transcript of the interview via e-mail for review to be sure their awareness of opioids had been correctly captured. Throughout the interview, notes were taken and kept with the transcriptions in the principal investigator's locked office. All audio files were stored on an external hard drive at the end of the interview sessions. Records of interviews were kept in separate files and labeled with a unique number.

\section{Data Analysis Procedures and Credibility}

Immersion into the data is one of the foundations of phenomenological research. ${ }^{8}$ The principal investigator was immersed in the data by conducting and transcribing all interviews, multiple readings, and taking notes during the process. These readings and rereadings identified emergent experiences as shared by the participants. These emergent experiences were labeled as codes that lead to the categories. The author finally organized the categories to gain a deeper understanding of the data. ${ }^{8}$ From these categories emerged themes that lead to the overarching meaning of the phenomenon. Dedoose (version 7.0.23; SocioCultural Research Consultants, Los Angeles, CA) was lastly used to organize the excerpts from the transcriptions according to themes. From reading the transcripts, coding, and categorizing that lead to themes, the principal investigator was able to understand the athletic trainers' awareness and practice of opioids and their patients.

Peer review and member checks were used as credibility strategies to ensure consistency and authenticity. ${ }^{8}$ Data were independently analyzed by one peer, qualitative researcher throughout the study, which included a review of the interview guide as well as validating codes. The peer used a general inductive approach that included identification of codes and themes. The peer followed the same inductive process as the principal investigator to uncover themes. The principal investigator and peer completed two consensus meetings throughout the analysis process to agree upon findings and determine if saturation was obtained. Member checking involved asking the participants to review transcript notes to confirm findings. ${ }^{8}$ After transcription, all research participants received via e-mail a copy of the interview transcripts for review, clarification, and suggestions. None of the participants in this study provided edits or corrections to transcripts.

\section{RESULTS}

Through data analysis, 3 common themes emerged: 1) athletic trainers have a responsibility to patients regarding opioids, 2) communication with patients about opioids, and 3) athletic trainers perceived they lacked education regarding opioids. Each theme is discussed in detail in the following section.

\section{Athletic Trainers Have a Responsibility to Patients Regarding Opioids}

As the opioid epidemic has spread, athletes are also at risk for misuse and abuse. ${ }^{1-6}$ All participants in this study agreed that athletic trainers have a responsibility to their patients concerning opioids. Athletic trainers having a responsibility was a theme that emerged to describe the belief that athletic trainers must either educate or monitor patients progress. Five of participants stated that as an athletic trainer, our role with opioids and the patient was educational and awareness. Participant 10 detailed this responsibility as educating athletes noting, "I think my role as a health care professional is to try and educate them and try and make sure they have some information about opioids." Participant 2 agreed that education was a responsibility as an athletic trainer and noted, "educating in a professional manner of what opioids do, and then how it creates an addiction." As a medical professional, athletic trainers play a crucial role in educating athletes and parents about safe opioid use and preventing opioid abuse.

Five of the participants in this study stated the role of an athletic trainer with opioids is monitoring the patient's progress. Often in collegiate and high school settings, the athletic trainer may see the patient daily after injury or surgery. As the participants stated in this study, athletic trainers can help monitor the patients' level of pain and use of opioids. Participant 1 spoke about the importance of monitoring the patient's progress stating, "I think our role is the progression of getting them back to a normal functioning state." Participant 3 mentioned the importance of monitoring and discussing their intake: "I encourage them to take their opioids as prescribed at least for the first few days before they start weaning off. Moreover, I will also have that conversation with their parents as an encouragement that this [opioids] is to help the body recover and reduce the stress on their body as they are recovering. I think there is a lot of hesitancy amongst athletes and parents about using opioids because they want to avoid the addictions that they hear about."

While none of the participants stated prescribing and administrating opioids was part of the athletic trainer's role, half of the participants stated it was essential to assist with monitoring the patients progress in pain management. As athletic trainers, we do have patients who may be in pain after an injury or surgery and as is shown with research on opioids and athletes, athletic trainers should be aware of potential use and abuse..$^{1-6}$

(C) The Internet Journal of Allied Health Sciences and Practice, 2020 


\section{Communication with Patients About Opioids}

When the participants in this study were aware that an athlete was taking opioids, most participants in this study stated they would communicate with their patients about opioids. Communication with patients about opioids was a theme that emerged to describe the belief that athletic trainers discuss pain management, addiction, and education about why the patient needed the medication. Athletic trainers should communicate with their doctors, coaches, athletes, and parents to assure patients understand proper opioid use. While this communication is not well outlined, athletic trainers can play a critical role through communication in the safe use, storage, and disposal of opioids.

While none of the participants in this study had a formal pain management plan, 8 out of the 10 participants did discuss levels of pain and taking opioids with their patients. Participant 7 addressed this communication as questioning the patient noting,

"I would check in and see how he was doing and how he was functioning and how he was getting along ... had he touched base with his professors? And then asked him how his pain was? How was his ability? How he was at night? Was he getting some sleep? Was he hurting as he was getting across campus?"

Participant 9 also stated the conversation would be more focused on moving away from prescription pain medication: "Let's try to get away from taking it during the day, you know or taking it prophylactically where you anticipate pain and maybe you just use it so you can make sure you can get to sleep, so you are getting enough rest, things like that." While many of the participants did not have a detailed discussion with their patients about opioids, Participant 10 instead had a very comprehensive discussion and was a private meeting in his office:

"I would have them come and talk to me in private and ask them about their pain control and what they were prescribed to take.... I would ask you what you were prescribed, and you would tell me whatever it is and then we would talk about dosage ... And then I would offer to them to take the minimal amount to get by particularly at night ... We would have a conversation about during the day you might be able to get away with just taking an over-the-counter medication, such as Ibuprofen or Tylenol. And at night is the time typically if you want to get a good night sleep particularly if you are hurting it is a good idea to maybe take your pain medication, and I would recommend that you try to take a half of one... a half-dose and see if that is enough to give you some comfort and relief."

Seven participants in this study educated the athlete about opioids. While one may presume that athletes are informed about opioids from either the pharmacists, nurses, or physicians, the patients still may have questions. An athletic trainer often interacts with the patients daily and can assist with dispelling any misunderstandings about opioids. Participant 10 stated that "I can educate them (athletes), you don't need to take this stuff every day. It is prescribed to take one pill every eight hours by mouth or every six hours and so we have a conversation about the education of opioids." Participant 10 also stated that in the conversation, he will educate them on the prescription: "You may not even finish what you have been prescribed. Because we are going to start exercising, and that in and of itself might make you start to feel well enough that you can just use over the counter [medications]. Just because you were prescribed pain medication doesn't mean you have to take them on the clock." Two of the participants (7 and 10) used the analogy of antibiotics and opioids stating "This (opioids) is not like an antibiotic; you do not take them until they are all gone."

Another common discussion with the patients was about opioids and addiction. The current emphasis in the news and public is that opioids are addicting. Two of the participants had previous experience with an athlete who was addicted to opioids while under their care. Four of the participants in this study did mention that they spoke to their athletes about addiction. When speaking on addiction Participant 5 stated: "There is a lot of hesitancy amongst athletes and parents about using opioids because they want to avoid the addictions that they hear about. I stress that when you have a patient that doesn't have a previous history of addiction, the likely hood of forming a habit in a week of opioid use is pretty low."

This investigator found that 8 out of the 10 participants discussed opioids with their patients. It was alarming to hear that 2 of the participants had no conversation with their athletes about opioids. As athletic trainers, we need to protect the patient and act in their best interest serving as an advocate for their welfare. As a health care provider, we must assist our patients through the health care system when at times the athlete may be trusting information from sources that are not as credible or not based on current knowledge or evidence-based guidelines.

\section{Athletic Trainers Perceived They Lacked Education}

All of the participants in this study stated they perceived themselves as uneducated about opioids. However, half of the participants have pursued ways to further their knowledge on opioids. Athletic trainers' education about opioids was a theme that emerged from the participant's discussion about needing to be further educated and perceived lack of knowledge about opioids. Education

(C) The Internet Journal of Allied Health Sciences and Practice, 2020 
of athletic trainers is defined as information offered by participants regarding their knowledge and understanding of opioids and their athletes. Five participants stated they had a pharmacology course in their athletic training education program while the other 5 participants never had a formal didactic course. Of the five participants who did have a pharmacology course in their athletic training education, they all could not recall explicitly addressing opioids. While participants in this study felt a lack of education about opioids as stated from the Board of Certification (BOC) Standards of Practice, an athletic trainer engages in lifelong, professional, and continuing education activities to promote continued competence. ${ }^{9}$

From this one study, it appears that 10 athletic trainers in District 10 perceived a lack of knowledge or education when it comes to opioids. Even though 5 of the participants in this study had a course on pharmacology, they still felt uneducated. Participant 2 spoke of about his educational background but noted minimal discussion on opioids: "We may have had a class on it . . . maybe one hour . . . but nothing substantial I certainly don't remember anything, maybe the red flags of opioid abuse." When addressing the pharmacology class, Participant 3 stated "It was very minimal We maybe spent a couple weeks ... it was just one of those things ... it wasn't something that as an athletic trainer we were going to be in direct contact with any sort of advising, prescribing, or dosage."

Half of the participants in this study received information about opioids after their education through their years of clinical practice. Participants 7, 8, and 9 stated their education on opioids has come from their experience and communication with other allied health care professionals. Participant 7 stated that his education was "informal just years of experience ... just talking ... talking to other athletic trainers, team physicians, stuff like that I've picked up along the way."

While the transition occurred years ago from the apprenticeship model, athletic trainers who were board certified over 15 years ago may not have had any formal education in the didactic setting regarding pharmacology. However, an athletic trainer must engage in lifelong, professional, and continuing education activities to promote continued competence. ${ }^{9}$ While five of the participants in this one study did not receive any formal education in their athletic training education about pharmacology, athletic trainers should maintain competence and up-to-date on current best-practice. As stated by Participant 10, "Even if you have the formal education, that doesn't mean that you know everything about it (pharmacology). You do yourself a disservice if you are not staying up-to-date. You always tell your students to be a sponge; well don't just tell them to be a sponge, be a sponge yourself. I have done that my whole career."

\section{DISCUSSION}

This study demonstrated that athletic trainers in District 10 who were working in a high school or collegiate settings felt that as a health care provider they had a role in sharing information about opioids to their patients. Athletic trainers are allied health care professionals that prevent, examine, diagnose, and treat acute or chronic injuries. ${ }^{9}$ Athletic trainers are highly qualified, multiskilled health care professionals who work in collaboration with an interprofessional health care team. One key role of an athletic trainer is the prevention of athletic injuries or conditions. As outlined in the BOC Standards of Professional Practice Standard 2: The Athletic Trainer implements measures to prevent and mitigate injury and illness. ${ }^{9}$ All participants in this study stated that athletic trainers have a role when it comes to opioids and our patients. However, participants were vague about this role in the expanding opioid crisis.

The participants in this study perceived a lack of education when it comes to opioids. Athletic trainers play a crucial role in providing patient education and promoting safe opioids use and preventing misuse and abuse. We are in a unique position to be informing our patients about appropriate and safe pain management strategies. However, as shown in this one study, if athletic trainers have minimal education on the subject, we may not be properly assisting our patients through the proper use of opioids.

Patients who have orthopedic surgical procedures may often require opioids for post-surgical pain. Pain control after an orthopedic procedure is essential for improved outcomes. Inadequate management of pain may result in both clinical and psychological changes that increase morbidity and mortality as well as costs and decreases the quality of life..$^{10} \mathrm{It}$ is alarming to note that the estimated economic burden of treating chronic pain that develops from acute pain in a 30-year-old individual over a lifetime could be as much as 1 million dollars. ${ }^{11}$ Post-surgical pain management is complex with several contributing factors that include inadequate opioid prescribing; system factors, such as fragmentation of care and lack of clear guidelines; and patient expectations. ${ }^{12-14}$ Effective strategies to assist with pain management postsurgical include multidisciplinary and collaborative care planning that includes patient input. ${ }^{15,16}$ Athletic trainers can play a critical role with patients who have postoperative pain and can assist with collaborative care and pain management. We can educate our patients and their families regarding postoperative pain. As was stated in the BOC blog, athletic trainers should initiate a dialogue about opioids with the athletes and parents if needed. ${ }^{17}$ Athletic trainers play a critical role in educating our patients and families about pain management.

(c) The Internet Journal of Allied Health Sciences and Practice, 2020 
While the purpose of this study was not assessing athletic trainers' knowledge of opioids, the participants in this study felt they lacked education about opioids, even those who had a pharmacology class through their athletic training education. While athletic trainers in this study may feel uneducated regarding opioids, it has been found that nurses also feel a lack in knowledge of important concepts, such as addiction, tolerance, dependence, pain assessment skills, and inaccurate knowledge of pharmacology and analgesics. ${ }^{18,19}$ Costello and Thompson found that nurses lacked in knowledge of safe storage and disposal ${ }^{18}$ In a study of orthopedic nurses, it was determined that nurses lack knowledge of opioid use in chronic pain conditions that may impair proper opioid administration as well as patient teaching for discharge. ${ }^{19}$ While the participants in this study identified a knowledge gap about opioids, ultimately the decision associated with the management of prescription medication is up to the physicians in consultation with the patient. Athletic trainers do have a responsibility to their patients to provide quality patient care and to act in the patients' best interest. ${ }^{9}$

Further research regarding athletic trainers' knowledge of opioids should be conducted and further explored. Due to the rising rates of opioid abuse and overdose deaths, it is vital that our patients be well informed about opioids. Research has demonstrated that patients want more information about pain management after surgical procedures. ${ }^{20}$ Horwitz et al also found that patients may not have a thorough understanding of their hospital discharge instructions. ${ }^{21}$ Kennedy-Hendricks et al reported that through a national survey on opioid practices, nearly half of the patients could not recall receiving information about safe storage or proper disposal of opioids. ${ }^{22}$ While nurses often address the patients' questions and concerns, research demonstrates there may be a gap in their knowledge, and educated, athletic trainers can help with this gap. Athletic trainers can assist patients in making informed decisions, and the patients should be educated about proper use of opioids, weaning off and storage of opioids, benefits and side effects, risks associated with opioids, the possibility of addiction, and disposal of unused opioids.

\section{LIMITATIONS AND FUTURE RESEARCH}

The findings discussed here show the small number of athletic trainers in one geographic area, limiting the generalizability of the findings. This small-scale qualitative study was exploratory in nature, and the results do not claim to be generalized to all athletic trainers. To fully understand athletic trainers' awareness and practice of opioids, studies should extend beyond the two settings and geographic area as examined in this study.

The purpose of this study was to investigate athletic trainers' perceptions, and participants stated they lacked education. While the principal investigator did not ask questions regarding their full educational background, the principal investigator cannot state they lacked education. Gathering more data about education and learning regarding opioids could provide additional value to studying this phenomenon of opioids and athletic trainers. To further explore opioids and athletic trainers' awareness and practice research should involve investigating, through quantitative analysis, athletic trainers' knowledge and management of patient's pain.

\section{CONCLUSION}

Research has demonstrated that high school students who use opioids before graduation are at an increased risk for future opioid misuse after high school. ${ }^{2}$ Research has also demonstrated that males who participate in organized sports have higher odds of being prescribed opioid medication and higher odds of past-year medical misuse. ${ }^{4}$ As health care providers, athletic trainers are in critical positions to educate patients on appropriate pain management and safe use of opioids. Participants in this study described a responsibility to athletes regarding opioids while also perceiving a lack of education as an athletic trainer. Through the data presented, athletic trainers may need to pursue continuing education on opioids to better assist their patients in making informed decisions. More research is needed to explore athletic trainers and the roles they play within an active population and opioids.

(C) The Internet Journal of Allied Health Sciences and Practice, 2020 


\section{REFERENCES}

1. McCabe SE, West BT, Boyd CJ. Leftover prescription opioids and nonmedical use among high school seniors: a multi cohort national study. J Adolesc Health. 2013;52(4):480-485. [PMID 23298996]

2. Miech R, Johnston L, O'Malley PM, Keyes KM, Heard K. Prescription opioids in adolescence and future opioid misuse. Pediatr. 2015;136(5):e1169-e1177. [PMID 26504126]

3. Veliz P, Epstein-Ngo Q, Austic E, Boyd C, McCabe SE. Opioid use among interscholastic sports participants: an exploratory study from a sample of college students. Res Q Exerc Sport. 2015; 86(2): 205-211. [PMID 25514090]

4. Veliz, P, Epstein-Ngo QM, Meier E et al Painfully obvious: A longitudinal examination of medical use and misuse of opioid medication among adolescent sports participants. J Adolesc Health. 2014;54(3):333-340. [PMID24225446]

5. Veliz PT, Boyd C, McCabe SE. Playing through pain: Sport participation and nonmedical use of opioid medications among adolescents. Am J Public Health. 2013;103(5):28-30. [PMID 23488520]

6. National Collegiate Athletic Association. NCAA Student-Athlete Substance Use Study: Executive Summary. 2014; Available at http://www.ncaa.org/about/resources/research/ncaa-student-athlete-substance-use-study-executivesummary- august-2014. Accessed June 1, 2018.

7. Health and Human Services. HHS Acting Secretary Declares Public Health Emergency to Address National Opioid Crisis. https://www.hhs.gov/about/news/2017/10/26/hhs-acting-secretary-declares-public-health-emergency-address-nationalopioid-crisis.html. Published October 26, 2017. Accessed December 14, 2018.

8. Creswell J. Qualitative Inquiry and Research Design: Choosing Among Five Traditions. Thousand Oaks, CA: Sage, 2005.

9. Board of Certification for the Athletic Trainer. BOC Standards of Professional Practice. Version 3.1. 2017; Available at: http://www.bocatc.org/system/document_versions/versions/144/original/boc-standards-of-professional-practice-201820180305.pdf?1520264560. Accessed June 1, 2018.

10.Carr DB, Goudas LC. Acute pain. Lancet. 1999;353(9169):2051-2058.

11.Cousins MJ, Power I, Smith G. Pain: a persistent problem. Reg Anesth Pain Med. 2000;25(1):6-21. [PMID 10660235].

12.Buck M, Paice JA. Pharmacologic management of acute pain in the orthopedic patient. Orthop Nurs. 1994;13(6):14- 23. [PMID 7870475].

13.Couling S. Nurses' and doctors' knowledge of pain after surgery. Nurs Stand. 2005;19(34):41-49. [PMID 15898252]

14.Miller EH, Belgrade MJ, Cook M et al Institution-wide pain management improvement through the use of evidencebased content, strategies, resources, and outcomes. Qual Manag Health Care. 1999;7(2):28-40. [PMID 10346460].

15.Gordon DB, Dahl JL. Quality improvement challenges in pain management. Pain. 2004;107(1):1-4. [PMID 14715381].

16.Cordts GA, Grant MS, Brandt LE, Mears SC. A qualitative and quantitative needs assessment of pain management for hospitalized orthopedic patients. Orthop. 2011;34(8):e368-e3373. [PMID 21815578].

17. Rotenberg D. Opioid abuse series part 2: opioid abuse risks after surgical intervention. BOC blog website. Available at: http://www.bocatc.org/blog/opioid-abuse-series-part-2-opioid-abuse-risks-after-surgical-intervention. Accessed June 2, 2018.

18.Costello M, Thompson S. Preventing opioid misuse and potential abuse: the nurse's role in patient education. Pain Manag Nurs. 2015;16(4):515-519. [PMID 25497402].

19. Mathews E, Malcolm C. Nurses' knowledge and attitudes in pain management practice. Br J Nurs. 2007;16(3):174- 179. [PMID 17363885].

20.Kastanias P, Denny K, Robinson S, Sabo K, Smith K. What do adult surgical patients really want to know about pain and pain management? Pain Manag Nurs. 2009;10(1):22-31. [PMID 19264280].

21. Horwitz L, Moriarty J, Chen C, et al Quality of discharge practices and patient understanding at an academic medical center. JAMA Intern Med. 2013;173(18):1715-1722. [PMID 23958851].

22.Kennedy-Hendricks A, Gielen A, McDonald E, McGinty EE, Shields W, Barry CL. Medication sharing, storage, and disposal practices for opioid medication among US adults. JAMA Intern Med. 2016;176(7):1027-1029. [PMID 27295629].

(C) The Internet Journal of Allied Health Sciences and Practice, 2020 